would be very useful for the computation of proper motions. It was especially urged that it should be indicated whether proper motions had been used to reduce the positions to a common epoch, and whether or not they had been corrected for magnitude equation.

Professor Dneprowsky gave a summary of the results obtained by Erthel's vertical circle at Pulkowo for the new fundamental catalogue for I925 of 1334 stars between the pole and $-10^{\circ}$ declination. From the upper and lower culminations a correction of $-.058^{\prime \prime}$ was deduced to the refraction constant of the Pulkowo tables. The corrections to Boss's declinations are in close agreement with those derived by Raymond.

M. Fayet stated that at Nice they are observing intermediary stars between $-15^{\circ}$ and $+15^{\circ}$ in three zones, of which two have been completely observed. The zone $-5^{\circ}$ to $+5^{\circ}$ is being printed and contains determinations of the proper motions of all the stars.

Mr Morgan stated that at Washington the intermediary stars were being observed between $-30^{\circ}$ and the North Pole. Dr Jones said that at the Cape stars down to the seventh magnitude were being observed between $-30^{\circ}$ and the South Pole, but these are not intermediary stars. Mr Morgan raised the question as to the number of fundamental stars required, whether the 750 proposed by $\mathrm{Mr}$ Stewart would be enough or whether it would be advisable to observe all 3000 of the Backlund-Hough list. A discussion took place on the difficulties of observing faint stars. Stars fainter than $8^{\mathrm{m}} \cdot 0$ are sometimes difficult to observe and the errors may often be greater than those of brighter stars. It was generally agreed that the meridian circle should furnish accurate positions of all stars down to $8 \mathrm{~m} \cdot \mathrm{O}$ and to fainter magnitude in those regions of the sky where the number of stars brighter was not sufficient for the reduction of photographic plates.

Dr Kopff stated that 14,000 stars were being observed in connection with the photographic revision of the A.G. zones. These are not intermediary stars but were chosen so as to give an average of ten on each plate.

Mr Dawson reported on the progress of the La Plata Observatory in the observation of 7800 stars between $-57^{\circ}$ and $-62^{\circ}$ completing their work on the zone $-52^{\circ}$ to $-66^{\circ}$.

Dr Moreau described a simplified graphical method for the reduction of meridian observations by the differential method.

$\mathrm{Mr}$ Sanders described a method which has been tried by him and Mr Raymond of measuring declinations by observing pairs of stars with zenith telescope and meridian circle on the same night. The two advantages of this method are that nadir observations are unnecessary and that half of the observations are made purely differential.

The other points raised in the report were dealt with briefly but it was not considered necessary to have any formal resolutions recommending them.

\title{
Commission 9. (INSTRUMENTS.)
}

M. Hamy, président, étant absent, M. Ch. Fabry a été désigné par le Comité exécutif pour le remplacer dans ses fonctions.

Ont pris part, en outre, aux travaux de la Commission: MM. de la Baume Pluvinel, Henri Chrétien, Delvosal, Nušl, Silva, Membres de la Commission; et MM. J. Baillaud, Esclangon, Evershed, Gregory, Innes, Van Heel, Lejay, Lismann, Nechvile, Sirks, Svoboda, Tinoco. 
Sur la proposition du président, la Commission désigne M. Chrétien comme Secrétaire.

La Commission prend pour base de ses travaux le Rapport rédigé par M. Hamy.

Sur la proposition de M. Fabry, les sujets à traiter seront examinés successivement d'après les rubriques suivantes: $A$. Matières d'optique; $B$. Grands instruments d'observation; C. Instruments auxiliaires et accessoires.

A. Matières d'optique. Les matériaux pouvant servir à la construction des miroirs plans ou courbes sont passés en revue: les métaux, le quartz, le verre.

Parmi les métaux qu'on pourrait utiliser, on indique: l'acier stainless, la stellite, l'invar, l'acier chromé et l'acier recouvert de chrome, comme particulièrement avantageux à cause de leur dureté, de leur inaltérabilité et de leur faible dilatation. L'invar est signalé comme instable. Malheureusement le pouvoir réflecteur de ces surfaces métalliques ne dépasse guère 60 per cent. Peut-être pourrait-on argenter les surfaces, comme l'a fait Melle Clavier*.

La silice fondue serait une substance idéale, à cause de son très faible coefficient de dilatation; mais on ne l'obtient que très difficilement en disque d'une certaine dimension: M. Esclangon dit qu'un disque de $20 \mathrm{~cm}$. de diamètre coûte dix mille francs.

M. Evershed attire à nouveau l'attention sur le Pyrex; mais il ne sait pas jusqu'à quelles dimensions on pourrait atteindre.

M. Chrétien résume les travaux récents du Professeur G. W. Ritchey. Par l'adoption des miroirs dits cellulaires qui ne font intervenir que des feuilles de glace bien homogènes, mesurant tout au plus $25 \mathrm{~mm}$. d'épaisseur, on pourrait vraisemblablement atteindre plusieurs mètres de diamètre. Avec ce genre de construction, le Pyrex peut être avantageusement employé et les Glaceries de SaintGobain se déclarent capables de fournir des disques de Pyrex de grand diamètre.

B. Grands instruments d'observation. Le Président demande à M. Chrétien de résumer l'état de la question des télescopes à deux miroirs formant un système aplanétique.

Après avoir rappelé les travaux théoriques de Schwarzschild et décrit la disposition préconisée par le regretté Directeur de l'Observatoire de Potsdam, M. Chrétien expose les origines du télescope Ritchey-Chrétien, élaboré au MontWilson en I9Io et décrit les avantages qu'il procure pour la construction des très grands instruments.

Grâce à l'observatoire de Paris et à son directeur d'alors, M. B. Baillaud, un modèle de $50 \mathrm{~cm}$. de diamètre a pu être terminé en 1927 . Ce modèle a permis de constater, d'une part, la très grande perfection optique des images sur toute l'étendue du champ; d'autre part, la possibilité de la réalisation de télescope du type Ritchey-Chrétien de très grand diamètre.

M. Chrétien ajoute que M. Couder étudie, de son côté, la réalisation des formes du type Schwarzschild, plus avantageuses encore au point de vue de la qualité optique des images, mais beaucoup plus encombrantes.

M. Van Heel demande si l'on peut avoir quelques explications sur la méthode d'examen de l'aberration sphérique de $M$. Couder dont il est question au Rapport. M. Chrétien en expose le principe et explique comment son emploi est bien adapté au contrôle de la taille des miroirs aux sections méridiennes transcendantes nouvelles.

- Des miroirs en acier CCR possèdent une remarquable stabilité et prennent un poli admirable. 
C. Instruments auxiliaires et accessoires. A la demande des membres de la Commission, M. Chrétien donne quelques explications complémentaires sur le procédé photographique à grande luminosité qu'il a décrit récemment aux Comptes-Rendus de l'Académie des Sciences de Paris.

M. Nušl a poursuivi l'étude du nouvel Astrolabe à prisme qu'il a présenté à la Commission au Congrès de Cambridge. Il résume les difficultés qu'il a rencontrées pour la mise au point du micromètre entraîné enregistreur. Dans ce micromètre, la compensation du mouvement des images stellaires est obtenue par le déplacement relatif de deux lentilles de puissances égales et de signes contraires, comme dans les micromètres des périscopes de marine.

Toutes les causes d'erreur ont pu être finalement découvertes et éliminées. Cette étude sera publiée très prochainement.

Les dimensions à donner au bain de mercure employé dans cet instrument et en général dans tous les instruments de haute précision où l'on doit recourir à la verticale, doivent être, d'après M. Nušl, notablement plus grandes que celles adoptées généralement.

M. Esclangon, consulté à propos des critiques formulées contre le bain de mercure confirme que, pour lui, c'est encore le meilleur instrument de référence à la verticale. M. J. Baillaud signale que M. R. Baillaud a étudié à Nice l'influence sur la surface libre du défaut d'horizontalité du fond.

Tout le monde est d'accord pour préconiser, en tout cas, l'emploi de bains épais.

Le rôle capital que joue le bain de mercure dans un grand nombre d'instruments de haute précision, incite la Commission, sur la proposition de $M$. Nušl à instituer dans son sein une sous-commission-n'ayant aucun caractère officieldite Sous-commission du Bain de Mercure. La présidence en est demandée à M. de la Baume Pluvinel, qui l'accepte et se charge de centraliser à Paris, tous les documents relatifs à cette importante méthode.

Font, en outre, partie de cette sous-commission: MM. J. Baillaud, R. Baillaud, Esclangon, Nušl, Tinoco.

Le Comité exécutif a renvoyé à l'examen de la Commission 9 deux questions présentées par la délégation polonaise, l'une concernant le prêt international de certains instruments; l'autre la création d'un "pied-à-terre" relevant de l'Union Astronomique.

En l'absence de leur promoteur, ces questions n'ont pu être discutées à fond.

Cependant, M. Innes signale à l'attention de la Commission la libéralité avec laquelle les observatoires de l'Afrique du Sud ont toujours accueilli les astronomes de tous pays désireux de travailler sous leur beau climat.

M. Innes passe en revue les principales stations astronomiques érigées dans 1'hémisphère austral par quelques Instituts américains. Il transmet ensuite une invitation, non officielle, émanant de la Ville de Kimberley et s'adressant à des astronomes pourvus de leurs instruments, les assurant de la plus grande attention dont ils seront l'objet et des facilités qui leur seront octroyées.

M. Gregory signale que l'Université de Londres vient de créer un nouvel observatoire, situé à Mill Hill, au nord de Londres. On espère pouvoir y commencer les travaux de recherches dans six mois. Il sera muni d'un télescope de $60 \mathrm{~cm}$. de diamètre, newtonien et avec amplificateur de Cassegrain, par Grubb; d'un cœlostat et d'un réseau de Rowland.

On se propose surtout d'y étudier les méthodes de recherches, et d'y éprouver les nouveaux instruments sur une assez grande échelle pour en dégager leurs possibilités quant à une mise en ouvre plus étendue. 
Les travaux sont poursuivis en collaboration avec les laboratoires de physique et de chimie de l'Université. Les étudiants peuvent y préparer leurs thèses.

Sur la proposition de M. Gregory, la Commission est unanimement d'avis d'intensifier son action et de maintenir son activité permanente-entre les Congrès. A cette fin, il est décidé, dès maintenant, que la Commission aura un centre permanent à Paris (à l'Institut d'Optique Théorique et Appliquée) et des centres de correspondance: en Angleterre, M. Gregory; en Europe centrale, M. Nušl; en France, M. Henri Chrétien; en Pays-Bas, M. Van Heel; en Amérique, à désigner (M. Pease (?)).

Le lendemain, mardi ro juillet, les membres de la Commission ont visité les laboratoires d'optique de la Marine néerlandaise, sous la conduite du Directeur, M. J. F. Sirks.

Résumant les travaux de la Commission des Instruments devant l'Assemblée générale de cloture, M. Fabry a dit, en substance:

La Commission 9 ne présente à l'Assemblée aucune proposition à adopter: son rôle est surtout celui d'information réciproque. Elle émet le vœu de recevoir de ses membres tous renseignements relatifs à l'activité instrumentale de leurs pays et se propose éventuellement de les publier.

\section{Commission 12. (Physigue Solatre.)}

The President, Dr C. E. St John, opened the first meeting of the Commission, and expressed the wish that the communications and discussions would be as interesting and fruitful as those held at Cambridge in $\mathrm{Ig25.}$

He asked Messrs Abetti, Butler and Minnaert to act as Secretaries of the meeting. At his proposal, an Advisory Committee, composed of Messrs Newall, Mitchell and Minnaert, was entrusted with the task of discussing with the President the working programme for the different meetings of the Solar Commission. Professor Brunner, Rev. Father O'Connor and Dr d'Azambuja were added as new members of the Commission. A centre for determining the index of solar activity was formed, composed of Messrs Abetti, d'Azambuja, Butler, Chapman, Evershed, and Rodés. Dr St John stated that this index had been desired by the Solar-Terrestrial Relationships Committee of the International Research Council, in order that geophysicists and others might have the means of investigating any possible correlations between various phenomena. The scheme formulated provided for a preliminary estimate by all observers of the various solar features, spots, faculae, bright calcium flocculi, bright and dark $\mathrm{Ha}$ flocculi.

The President then addressed the visitors present, bade them welcome, and expressed the hope that they would freely take part in all the discussions. At the request of the President, the directors of the different centres for solar investigations gave a short account of the work done at their Institutes during the last three years. The following gentlemen successively gave such an account: Rev. Father Rowland and Rev. Father O'Connor (Stonyhurst Obs.), Mr Butler (for Greenwich), Dr St John (Mt Wilson Obs.), Professor Brunner (Zurich), Rev. L. Rodés (Madrid), Professor Abetti (Arcetri), Mr Evershed (Ewhurst), Professor Newall (Cambridge), Professor Deslandres and Dr d'Azambuja (Meudon), Professor Da Costa Lobo (Coïmbra) and Dr Freundlich (Potsdam).

Beautiful spectroheliograms of the sun's disc in the light of an infra-red calcium 